\title{
Farnesol-Containing Macromolecular Systems for Antibiofilm Strategies
}

\author{
Maria Nowacka ${ }^{1, *} \mathbb{D}$, Anna Kowalewska ${ }^{1}\left(\mathbb{D}\right.$ and Dorota Kręgiel ${ }^{2}(\mathbb{D})$ \\ 1 Centre of Molecular and Macromolecular Studies, Polish Academy of Sciences, Sienkiewicza 112, \\ 90-363 Łódź, Poland; anko@cbmm.lodz.pl \\ 2 Department of Environmental Biotechnology, Lodz University of Technology, Wólczanska 171/173, \\ 90-924 Łódź, Poland; dorota.kregiel@p.lodz.pl \\ * Correspondence: mnowacka@cbmm.lodz.pl; Tel.: +48-42-680-32-03
}

Received: 12 May 2020; Accepted: 18 May 2020; Published: 21 May 2020

\begin{abstract}
Farnesol is a natural sesquiterpenoid and an interesting quorum-sensing molecule. Its insolubility in water is the biggest obstacle to its application for bacterial biofilm treatments since it compromises the bioavailability. Recently, an increasing interest in farnesol encapsulation or loading in polymeric materials may be noted due to the prolonged action of the active macromolecular systems. In this short review, we present an overview of methods leading to improved interactions between farnesol and microbial biofilms.
\end{abstract}

Keywords: farnesol; antibiofilm; quorum-sensing; adjuvant; polymers; encapsulation

\section{Introduction}

Biofilms that cover both abiotic and biotic surfaces provide a protective environment for the efficient growth of pathogenic bacteria and fungi. A critical factor for the optimal existence of microorganisms within biofilms is proper communication between the microbial cells. It ensures the synchronization of the morphological changes, growth, gene expression and secretion of compounds crucial for the colony development. In heterogeneous microbial biofilms, often one group of consortium utilize secreted signaling chemical molecules to coordinate collective behavior [1]. The cell-to-cell communication system, called quorum sensing (QS), involves numerous molecules that regulate biofilm development and, in consequence, surface colonization [2]. QS inhibitors (QSI) have been proposed as promising antibiofilm agents and therapeutic alternatives and/or adjuvants to currently failing antibiotics. QSI inhibit virulence factors essential for establishing infection and pathogenesis through targeting non-essential metabolic pathways [3]. The effect of naturally isolated plant compounds, non-toxic to the natural environment, on the repression of QS systems seems to be a very interesting alternative for disinfectant/antibiotic treatments. Especially, novel antimicrobial systems eliminating multiantibiotic-resistant biofilms are of increasing interest.

Farnesol (3,7,11-trimethyl-2,6,10-dodecatrien-1-ol) and its derivatives have an important role in the signal transmission between plants and other organisms, including plant pathogens: aphids and molds [4,5]. In microorganisms, farnesol is known as a quorum-sensing molecule, that can significantly increase extracellular polymeric substances (EPS) production by promoting polysaccharide biosynthesis [6]. Its chemical and biological activity has been recently reviewed [7]. Although the terpenoid is an important bioactive agent, the direct bioapplication of this compound is limited by its insolubility in water. Nanoencapsulation of essential oils in nanostructured systems has been employed in order to overcome their chemical instability and to improve their bioavailability under controlled release conditions [8]. The design of such high-performance multifunctional materials with a good activity against drug-resistant pathogens is often bio-inspired, mimicking biological processes and 
natural materials [9]. The encapsulation of farnesol in polymer nanoparticles and micelles or loading it into polymer gels improves both its stability and bioavailability. In this review, we present a summary of recent reports on macromolecular systems containing farnesol and its derivatives as antibacterial and antifungal agents as well as an auxiliary drug in skin reparative therapies and transdermal treatment.

\section{Farnesol and Its Antibiofilm Activity}

Chemically, farnesol (FOH) is an acyclic sesquiterpene alcohol (Scheme 1), endogenously synthesized via the ergosterol pathway, present in microbial, plant and mammalian cells. This compound is a major component of various plant essential oils, e.g., lily of the valley (Convallaria majalis), citrus (Citrus sp.) and nutmeg (Myristica fragrans). In mammalian cells, FOH is a key intermediate in de novo synthesis of cholesterol [10]. Farnesol, non-toxic to the natural environment, can be a very interesting alternative for disinfectant/antibiotic treatments, which are often ineffective and induce an increase in the microbial resistance.<smiles>CC(C)=CCC/C(C)=C/CC/C(C)=C/CO</smiles>

Scheme 1. Chemical structure of farnesol (3,7,11-trimethyl-2,6,10-dodecatrien-1-ol).

Farnesol is a quorum-sensing molecule secreted by the opportunistic human pathogenic yeast Candida albicans [11]. However, the mechanism behind its action is not completely understood [1]. It was speculated that $C$. albicans may exploit the genes regulating filamentation as a self-regulatory mechanism to induce yeast budding in mature biofilms, their dispersal and seeding of new niches. Due to the accumulation of farnesol, dense cultures of C. albicans have been reported to display a reduced tendency for the yeast-to-hyphal switch, inferring the role of $\mathrm{FOH}$ in inhibiting hyphae formation [12]. Farnesol's action as a QSI for C. albicans appears to be highly specific. Only E,E-isomer acts as a quorum-sensing molecule and supresses filamentation [13]. The morphological response to FOH in C. albicans seems to be also sensitive for minor structural changes in the molecule morphology $[13,14]$. It hints that a target-specific protein is most probably involved in its action. Farnesol can be transported over the bacterial membrane and targets several factors involved in the regulation of morphogenesis in C. albicans (adenylyl cyclase Cyr1, suppression of mitogen activated protein (MAP) kinase pathway and Ras1-cAMP signalling pathway) $[1,15,16]$. In addition, it was found that the removal of the C. albicans gene EED1, which governs hyphal maintenance, led to hypersensitivity to FOH as well as to its increased production. It suggests that there is a link between elongated hyphal growth and FOH signalling mediated via a specific sensor/receptor [17-19]. Farnesol may also induce cell death (apoptosis) by unspecific effects, e.g., membrane disturbances or glutathione extrusion [1]. C. albicans has evolved mechanisms that allow tolerating its high concentration in the environment. Despite being continuously released into the environment at a high cell density, it blocks filamentation, but cannot inhibit the elongation of already existing hyphae. Therefore, cells are sensitive to farnesol only for a limited time [20].

Farnesol affects many stages of biofilm development [21] and is responsible for the activation of extracellular traps' formations [14]. The expression of genes responsible for cell wall maintenance, cell surface hydrophilicity, drug resistance, iron transport, hyphae formation and heat shock proteins, e.g., TUP1 (general transcriptional corepressor 1 gene), CRK1 (serine/threonine-protein kinase 1 gene), PDE2 (phosphodiesterase 2 gene), FCR1 (fluconazole resistance 1 gene), PDR16 (phosphatidylinositol transfer gene), CHT2 and CHT3 (chitinase 2 and 3 genes) and CSH1 genes (cell surface hydrophobicity) is affected in farnesol-treated biofilms [22]. The activity of the FTR2 enzyme (formylmethanofurantetrahydromethanopterin formyltransferase), as well as the heat shock proteins' production (HSP70, HSP90, HSP104, CaMSI3, SSA2) are increased in the presence of FOH [22]. Farnesol increases C. albicans 
susceptibility to fungicides by influencing the expression of $C Y R 1$ (adenylate cyclase gene) and PDE2 (moderates signalling by cyclic adenosine monophosphate-cAMP) [23].

Farnesol takes part in interspecies communication [24]. This molecule formed by C. albicans may also inhibit biofilm formation and compromise the cell membrane integrity of methicillin-resistant and -susceptible strains of Staphylococcus aureus [25]. It has been recently shown that FOH-sensitive S. aureus lose the staphyloxanthin pigment, an important virulence factor [26]. Farnesol exposure caused oxidative stress in S. aureus cells is demonstrated by alterations in the redox sensors and major virulence regulators. Theoretical modelling indicated that $\mathrm{FOH}$ may block staphyloxanthin biosynthesis via competitive binding to the CrtM enzyme, owing to their high structural similarity. Moreover, farnesol significantly inhibited biofilm formation by Streptococcus mutans [27] and S. epidermidis [28]. It also interferes with the growth of $C$. neoformans, Penicillium expansum and affects the cell wall and cytoskeletal integrity in A. fumigatus [29]. Farnesol leads to the downregulation of the Pseudomonas aeruginosa quinolone signal and enables the coexisting of a mixed heterogeneous population of C. albicans and P. aeruginosa [30]. It also enhanced the permeability of S. aureus and E. coli to exogenous chemical compounds [31] and induced apoptosis in Aspergillus nidulans [32] and Aspergillus flavus [33]. The impact of $\mathrm{FOH}$ on human innate immune cells was also investigated [34].

The phenomenon of QS inhibition by farnesol has a big potential for the efficient reduction of gene expression. However, the demonstrated antibiofilm potential of $\mathrm{FOH}$ needs to be further explored in order to develop new therapeutic strategies against biofilm-associated infections. Its hydrophobicity and limited solubility in water reduces largely the bioavailability and, as a consequence, the antibiofilm activity on contaminated surfaces. Farnesol oils and stock solutions should be stored under a controlled atmosphere. Despite its long-term stability in water [11], the composition of the growth medium may be of importance for the $\mathrm{FOH}$ decomposition rate [35]. The encapsulation of farnesol in macromolecular materials may protect the hydrophobic molecules and help in their dispersion in aqueous systems enhancing their contact with biofilms. This approach may be an efficient solution for the multiple drug resistance in microbial pathogens. Such combinations of active drugs and polymeric materials offer important advantages over conventional antibacterial and antibiofilm systems: prolonged activity, non-volatility, chemical stability and reduced risk of developing resistance to active agents [36-40].

\section{Farnesol-Containing Polymer Materials}

Farnesol interactions with macromolecular systems can be found in nature. It was shown that odorant binding proteins (OBPs) of olfactory roles, present in insects such as Spodoptera exigua, exhibited considerable affinities to host odorants of acetophenone, farnesol and $\beta$-ionone $(\mathrm{Ki}<20 \mu \mathrm{M})$ [41]. Farnesol molecules can also be applied for the preparation of synthetic macromolecular systems, e.g., dendrimers that are interesting materials of unique architecture and applicative potential in medicine, biology and biotechnology. Well soluble carbosilane dendrimers (first to fourth generation) were obtained with high yields through the reaction between $\mathrm{FOH}$ and Si-Cl-bonded carbosilane parent dendrimers [42]. Farnesol and its derivative farnesyl were also applied as crosslinkers for polymeric sulfur (polymeric materials with $>50 \mathrm{wt} \%$ elemental sulfur as their major component) [43]. The application of $\mathrm{FOH}$ allowed the preparation of more rigid polymers with high $T_{\mathrm{g}}$. The foamed S-farnesol material exhibited extremely high affinity for mercury, making it a promising, low-cost material for an efficient $\mathrm{Hg}$ capture.

Farnesol derivatives can also be used as additives to improve properties of polymers. For example, farnesyl 3,5-di-tert-butyl-4-hydroxybenzoate and farnesyl 3-(3,5-di-tert-butyl-4-hydroxyphenyl) propionate were proved to influence the oxidation of a polyolefin elastomer [44]. The radical grafting of these compounds not only prevented their migration from the polymer matrix, but also enhanced the material's resistance to oxidation. The material can be applied for novel antioxidant active packaging, which are of improved long-term stability and attractive physicochemical properties. It should be stressed that the oxidation of lipids and microbial growth is the main cause of food spoilage [45]. The migration of low molecular weight antioxidant compounds and their extraction by liquid media is typically a significant 
disadvantage, which results in the deterioration of the properties of polymeric materials and the release of applied stabilizers [46]. Another farnesol derivative (3,7,11-trimethyl-2,6,10-dodecatrien-1-diaminobutane amide) was covalently incorporated into polyurethane (PU) chains resulting in the formation of a new type of polyurethane scaffolds (PU-F) [47]. It influenced the degradation, surface and mechanical properties of PU. Scaffolds for tissue engineering should be biocompatible, biodegradable, stimulate blood vessel ingrowth and have mechanical properties consistent with the anatomical site into which it is to be implanted [48]. The prepared material exhibited good biocompatibility, a capability to vascularize after implantation and an unconfined compressive of stiffness [49]. No inflammatory reaction was observed after the scaffold implementation and the modification positively influenced the cell-material interactions in bovine chondrocytes tests, but had no effect on cells' viability or proliferation.

Quite importantly, farnesol can be also used as an additive that reduces the rate of bacterial biofilms' growth on polymeric systems. In vitro experiments showed that FOH can act as an adjuvant with conventional antibiotics. For example, farnesol and lipopeptide from Bacillus subtilis AC7 (AC7BS) exhibited a synergistic effect on the inhibition of Candida albicans' biofilms' growth on catheters made of silicone elastomers [50]. C. albicans are responsible for dangerous infections caused by their biofilm formation, whereas $\mathrm{FOH}$ inhibits the filamentation regulating the yeast-to-mycelium conversion and thus influences the biofilm size [51]. The simultaneous application of both compounds exhibited a stronger activity than each molecule on their own. Their synergistic effect reduced $C$. albicans' adhesion up to $74 \%$ at $1.5 \mathrm{~h}$ and the biofilm growth up to $93 \%$ at $24 \mathrm{~h}$. Farnesol was also applied for the surface modification of orthopedic implants [52]. A limited blood flow and high porosity of bones increase the risk of bacterial infection in tissues surrounding implants. A high concentration of antibiotics that are required after orthopedic surgeries can produce irreversible organ damage. To prevent this situation, the simultaneous local delivery of an antibiotic (vancomycin) and an adjuvant (farnesol) was proposed to potentiate the therapeutic effect. Although the incorporation of antibiotic and hydrophobic $\mathrm{FOH}$ into a biocompatible and highly porous silica sol-gel was not a simple task, it did not influence the stability of the thin films on the surface of implants made of titanium alloy and anodized Kirshner wires, and neither did it affect the release of vancomycin. A complete inhibition of S. aureus was obtained for films composed of $30 \mathrm{wt} \%$ of farnesol and $10 \mathrm{wt} \%$ of vancomycin (around $10^{6}$-fold reduction in growth), whereas the application of identical amounts of the pure compounds had no bactericidal effect (Figure 1).

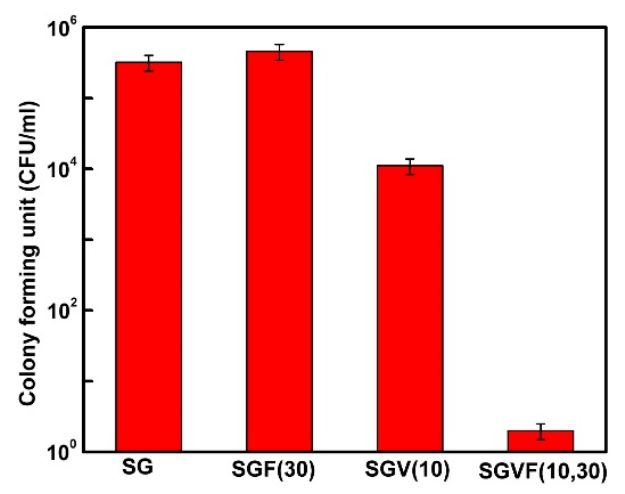

Figure 1. The anti-methicillin-susceptible S. aureus growth effect of thin sol-gel films containing vancomycin and farnesol, coated on Ti rods [52]. Reprinted with permission from (Biomaterials). Copyright (2014) Elsevier.

The suggested mechanism of action is based on a combined interaction of vancomycin and farnesol with bacterial cell walls and cell membranes (Figure 2). The antibiotic forms hydrogen bond with the terminal D-alanyl-D-alanine moieties of the peptides built of N-acetylmuramic acid (NAM) and $\mathrm{N}$-acetylglucosamine (NAG), thus preventing the proper formation of the cell wall and making it more prone to lysis $[53,54]$. However, penetration of the cell wall with the hydrophilic and highly protein 
bound vancomycin is difficult. Farnesol is able to penetrate the biofilm and enhances the permeability of bacterial cells to some chemical compounds (including vancomycin [55]) and its presence may help to reduce the number of adherent bacteria. Its hydrophobic molecules can enhance non-specifically the permeability of bacterial cells by accumulating in the cell wall and cell membrane, increasing its porosity within the cell wall and preventing its reparation mechanisms. It leaves a pathway for a vancomycin penetration of the cells.

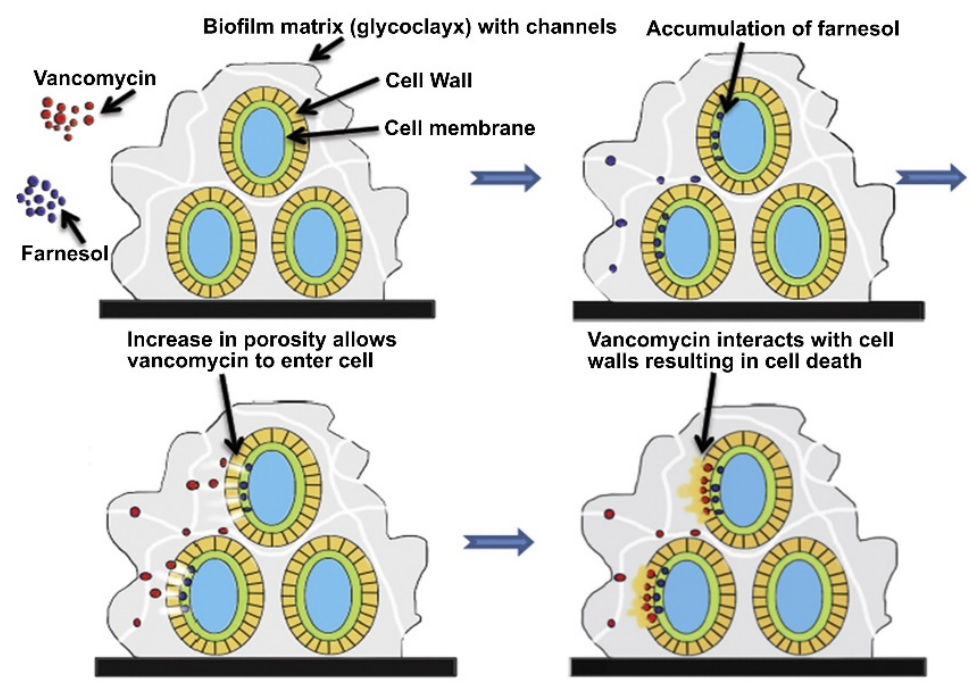

Figure 2. Farnesol adjuvant activity leading to the enhancement of vancomycin's effectiveness [52]. Reprinted with permission from (Biomaterials). Copyright (2014) Elsevier.

Molecules of farnesol, due to their amphiphilic structure, are capable of the formation of large supramolecular systems. It was shown that FOH-based heterocyclic cationic surfactants, [1-methyl3-(2-oxo-2-((farnesyl)oxy)ethyl)-1H-imidazol-3-ium bromide, 3-hydroxy-8-methyl8-(2-oxo-2-((farnesyl) oxy)ethyl)-8-azabicyclo[3.2.1]octan-8-ium bromide and 1-(2-oxo-2-((farnesyl)oxy)ethyl)pyridin-1-ium bromide)], can adsorb at the water-air interface and self-aggregate into micelles in aqueous systems [56]. Intramolecular cation $-\pi$ interactions, hydrolysis and molecular rearrangements resulted in the release of volatile compounds. Their type was influenced by the place where they were formed (inside micelles or at the air-water interface). Molecules of farnesol can also be loaded inside micelles, nanoparticles and liposomes formed by various polymers, such as poly(lactic acid), poly(methyl methacrylate) and polystyrene microparticles [57]. Such an encapsulation may also robustly improve its dispersion in other systems, including its aqueous solubility. It is also a very good solution to increase the stability of antibacterial and antifungal activity of $\mathrm{FOH}$ in the treatment of biofilms and planktonic cells.

Farnesol can also be encapsulated in silica capsules with oil cores obtained by a sol-gel method (water-in-oil-in-water- $\mathrm{O} / \mathrm{W} / \mathrm{O}$, multiple emulsions) using tetraethoxysilane as their precursor $[58,59]$. Amorphous silica-based capsules are an interesting alternative to organic-based micelles due to their good thermal stability and biocompatibility. This type of nanoparticles can find an application in cosmetics as an efficient but not expensive antioxidant-releasing material.

\subsection{Nanoparticles and Liposomes Containing Farnesol}

\subsubsection{Antibacterial Materials}

Nanoparticles can act on bacterial cells in biofilms and their components through peptidoglycan damage, plasmid nicking, oxidative damage to cell components by reactive oxygen species, enzyme disruption or protein denaturation [60,61]. Strategies involving nanoparticles include contact killing through cell membrane breakage, biofilm penetration through water channels and EPS, antimicrobial cargo loading, photosensitizer loading for photothermal effects or ion release [62]. Apart from the size 
and shape of nanoparticles, also their surface properties and interior structure (liposomes, micelles, dendrimers, mesoporous silica particles) may be tailored for the best effect.

For example, pluronics-formulated farnesol was used for efficiently killing Streptococcus mutans biofilms that are a causative agent of dental caries. A tooth-binding, negatively charged micellar drug delivery platform was designed using biocompatible Pluronic copolymers that encapsulated antimicrobial FOH [63]. Their functionalization with biomineral-binding alendronate moieties allowed an effective and swift binding ( $<1 \mathrm{~min})$ to the model tooth surface-hydroxyapatite (HAp-the main component of enamel) — and gradually released the encapsulated farnesol. In vitro biofilm inhibition studies demonstrated that they were able to provide a significantly stronger inhibition of Streptococcus mutans' biofilm formation on HAp discs than the untreated blank control micelles $(p<0.0001)$. Other studies showed that the biomass was significantly decreased (SNK test, $p<0.05$ ) in the presence of FOH encapsulated in Pluronics micelles, relative to untreated biofilms [64]. The formation of large towers containing dead cells appeared to be specific to formulated farnesol (this phenomenon was not observed in the presence of triclosan). The cell killing was independent of the tower formation. Parallel $\mathrm{CFU} / \mathrm{mL}$ tests revealed that biofilm growth in the presence of Pluronic-formulated FOH resulted in a 3-log reduction in viability, whereas the viability in the presence of neat farnesol decreased by less than 1-log. The Pluronics-formulated FOH was able to alternate the biofilm architecture, presumably via interactions with the sucrose-dependent biofilm matrix.

The antibacterial efficacy of formulations containing farnesol can also be improved by the application of $\mathrm{pH}$-responsive polymer nanoparticle carriers (NPCs) which may be of importance in acidic microenvironments of bacterial biofilms. Such an approach to drug delivery enhanced the therapeutic efficacy and reduced the side effects $[65,66]$. Stimuli-responsive materials can be designed to modify their features in response to the nature of the microorganisms (changes in $\mathrm{pH}, \mathrm{pO}_{2}$, temperature, bacterial-secreted compounds) in order to improve their performance against infections and biofilms [67]. Antibacterial $\mathrm{pH}$-responsive nanoparticles become a means for controlling oral biofilm-related infections during various stages of biofilm formation $[68,69]$. FOH release from nanoparticles as a function of $\mathrm{pH}$ is a novel, promising way to inhibit biofilm formation and reduce the number and severity of carious lesions. Farnesol-loaded p(DMAEMA)-b-p(DMAEMA-co-BMA-co-PAA) [poly(dimethylaminoethyl methacrylate)-b-poly(dimethylaminoethyl methacrylate)-co-butyl methacrylate-co-propylacrylic acid] micelles can be successfully applied against $S$. mutants' biofilm formation $[70,71]$. FOH-containing nanoparticles capable of binding to hydroxyapatite (HA), saliva-coated HA (sHA) and exopolysaccharides with an enhanced drug release at an acidic $\mathrm{pH}$ were developed [70]. It was loaded into cationic nanoparticles formed by diblock copolymers composed of 2-(dimethylamino)ethyl methacrylate (DMAEMA), butyl methacrylate (BMA) and 2-propylacrylic acid (PAA) (p(DMAEMA)-b-p(DMAEMAco-BMA-co-PAA)) (Figure 3). The cationic nanoparticles adsorbed very well onto the negatively charged HA, sHA and exopolysaccharide-coated sHA due to the strong electrostatic interactions via the multivalent tertiary amines of $\mathrm{p}$ (DMAEMA). FOH was loaded into hydrophobic cores at $\sim 22 \mathrm{wt} \%$. Farnesol release was found to be $\mathrm{pH}$-dependent with $\mathrm{t}_{1 / 2}=7 \mathrm{~h}(\mathrm{pH} 4.5)$ and $15 \mathrm{~h}(\mathrm{pH} 7.2)$. At an acidic $\mathrm{pH}$ (characteristic of cariogenic biofilm microenvironments), the nanoparticles underwent core destabilization. Topical applications of the FOH-loaded nanoparticles disrupted biofilms of Streptococcus mutans four times more effectively than free farnesol and reduced both the number and severity of carious lesions (free FOH had no effect). Treatment drug-loaded nanoparticles compromised the mechanical stability of the biofilms compared with the free farnesol and control experiments ( $>2 \times$ enhancement in the biofilm removal under shear stress). 


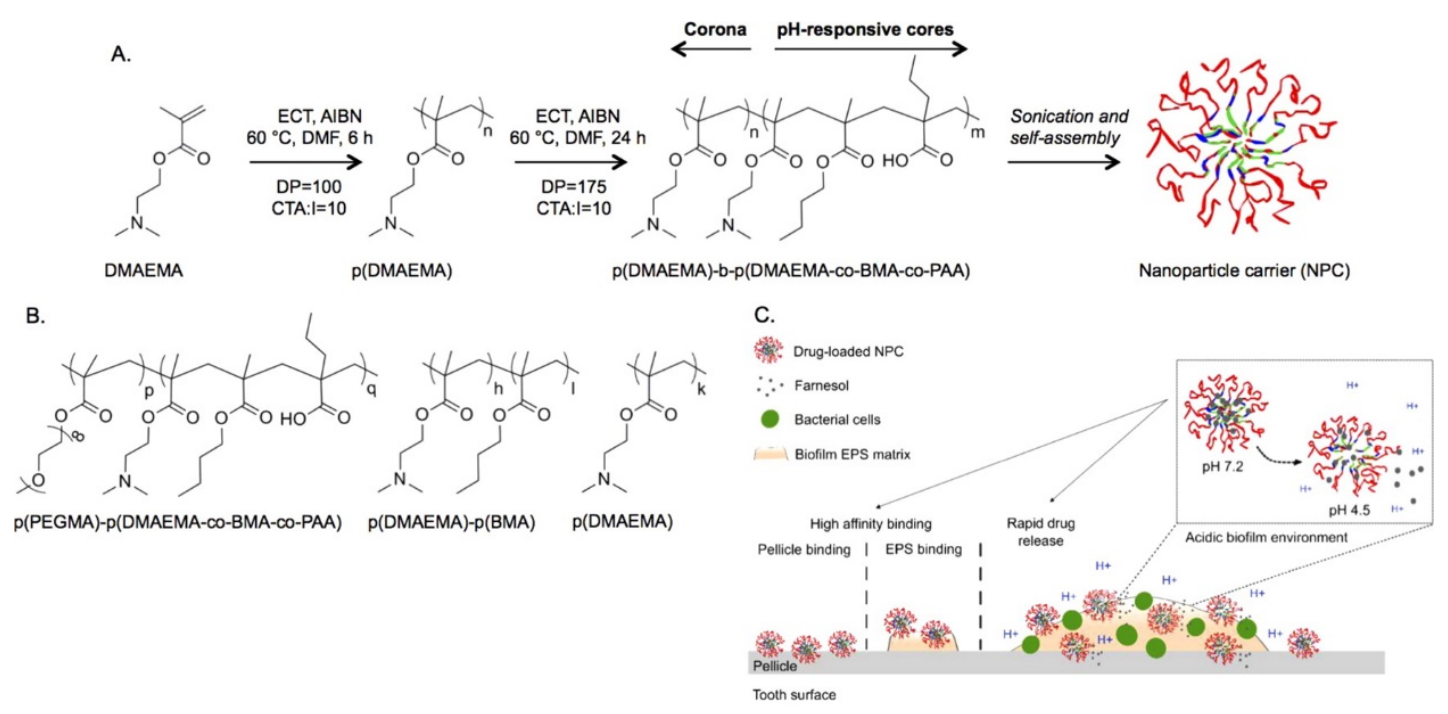

Figure 3. Synthesis and function of polymeric nanocarriers of farnesol and their antibiofilm activity [70]. (A) Depiction of the chemistry and self-assembly of diblock copolymers. (B) Structures of control polymers utilized to isolate the required physicochemical characteristics for binding to dental surfaces. (C) Proposed mode of action of $\mathrm{pH}$-responsive nanoparticles for the prevention and/or treatment of biofilms. Reprinted with permission from (ACS Nano). Copyright (2015) American Chemical Society.

Another way to prepare antimicrobial materials is by an application of $\mathrm{FOH}$ derivatives, e.g., farnesal (3,7,11-trimethyl-2,6,10-dodecatrienal) [72]. It exhibits similar anti-bacterial properties against $S$. mutans, and is also highly hydrophobic. A novel drug delivery system was obtained by the solubilization of farnesal through linking it to polyethylene glycol chains and conjugation with tris(tetra- $n$-butylammonium) hydrogen pyrophosphate. This procedure allowed the synthesis of polymeric micelles that were able to rapidly bind to hydroxyapatite and remain there for up to $12 \mathrm{~h}$. Nearly $90 \%$ of the encapsulated farnesal was released within $30 \mathrm{~min}$ in an acidic $\mathrm{pH}$ environment $(\mathrm{pH}=4.5)$, suggesting that the release takes place selectively under acidic conditions.

It is known that saturated conditions enhance drug loading [73]. The application of the saturated FOH solution improved the drug loading capacity and prolonged the time with a zero-order drug release to $48 \mathrm{~h}$. Farnesol-loaded NPCs, varying in the overall molecular weight, and corona-to-core molecular weight ratios were also used [74]. The active molecules were located in the $\mathrm{pH}$-responsive core of diblock co-polymer NPCs synthesized by the sequential copolymerization of 2-(dimethylamino)ethylmethacrylate (DMAEMA), butyl methacrylate (BMA) and 2-propylacrylic acid due to hydrophobic interactions. NPCs loaded at saturated conditions exhibited $\sim 300 \%$ greater drug loading capacity over standard conditions. A zero-ordered drug release was noted over $48 \mathrm{~h}$ (three times longer than standard farnesol loading). Their anti-biofilm activity towards planktonic Streptococcus mutans and their biofilms was markedly amplified. The total biomass was reduced by disrupting the insoluble glucan formation and increased the NPC cell membrane localization.

Antimicrobial properties against S. mutants were also exhibited by FOH-loaded halloysite nanotubes (Fa-HNT) in dental resin composites [75]. Halloysite nanotubes are two-layered alumino-silicate tubular structures [76]. Their empty interior can be used as a potential carrier for entrapped drugs [77]. Fa-HNT were incorporated in flowable resin composites consisting of methacrylate monomers and dental glass fillers at concentrations of 1-20\% (wt./wt.). The addition of Fa-HNT caused an enhancement of the compressive strength as well as the flexural modulus of the composite, but decreased the flexural strength. The dental composite exhibited a significant antibacterial activity with an increase in the area of the zone of inhibition against the strains of Streptococcus mutans. The Fa-HNT resin composites were not cytotoxic (tests on NIH-3T3 mouse embryonic fibroblast cell lines) and may be used as multipurpose restorative dental materials. Farnesol was also used as an upgrading additive to a glass ionomer cement (GIC-a restorative material) [78]. The incorporation of FOH into GIC inhibited the growth of the cariogenic 
bacteria S. mutans but had a little effect on the composition, structure and physiology of the biofilm matrices. Larger zones of inhibition $(p<0.05)$ were noted and the polysaccharides levels increased over time. Bacterial viability in biofilms was reduced for a short term, yet the biomass was similar $(p>0.05)$. Moreover, the GIC's hardness was increased and it showed greater biocompatibility to human cell HaCaT.

Farnesol can be applied along with other antibacterial agents. For example, the biofilm anti-adhesion activity of poly(vinyl alcohol)-coated silver nanoparticles (AgNPs-PVA) and FOH was evaluated using bovine root dentine [79]. The minimum inhibitory concentration (MIC) and minimum microbicidal concentration (MMC) of the AgNPs-PVA and farnesol were evaluated against E. faecalis $(42.5 / 50 \mu \mathrm{M}$ and $0.85 / 1.0 \%$, respectively), C. albicans $(27.5 / 37.5 \mu \mathrm{M}$ and $1.75 / 2.5 \%)$ and P. aeruginosa (32.5/32.5 $\mu \mathrm{M}$ and 2.5/2.75\%). The antimicrobial and biofilm anti-adhesion activities of AgNPs-PVA and farnesol show their potential for use as a co-adjuvant in endodontic treatment (auxiliary procedure for root canal disinfection) as well as intracanal medications, root canal sealers and/or repair cements.

\subsubsection{Antifungal Materials}

Nanostructured farnesol may be a promising alternative to conventional antifungal therapies, including systems of synergistic action with other drugs. Farnesol as a fungal quorum-sensing molecule (QSM) was co-delivered with antibiotic ciprofloxacin as a liposomal formulation to eradicate Pseudomonas aeruginosa biofilms [80]. It was proved to be a promising approach that gives better results in biofilm killing than the application of the free antibiotic. The liposomes were prepared using the dehydration-rehydration method. This antibiofilm strategy synergistically combined the microbial quorum-sensing and bactericidal agent's delivery and led to superior biofilm killing (Figure 4). The efficacy of liposomes and the detrimental effects of $\mathrm{FOH}$ on P. aeruginos a were assessed using a standard biofilm assay. It was shown that the ciprofloxacin release from liposomes was higher when encapsulated with farnesol (Lcip + FOH) compared with the antibiotic alone, whereas the FOH release was lower for Lcip + FOH compared with liposomes containing only farnesol. The biofilm metabolism was significantly lower when it was simultaneously treated with Lcip + FOH or Lcip compared with free ciprofloxacin. The biofilm disruption was confirmed for the treatment with Lcip $+\mathrm{FOH}$, with a greater dead cell ratio and increased depth of biofilm destruction than with other liposomal preparations. The addition of $\mathrm{FOH}$ allowed the application of a lower concentration of ciprofloxacin. It should be noted that typically a drug penetration into dense P. aeruginosa biofilms is difficult and results in an ineffective infection clearance.

Farnesol was also used to cure vulvovaginal candidiasis (VVC) caused by the opportunistic fungus Candida albicans, whose yeast to hyphae transition is considered a major virulence factor [81]. A mucoadhesive nanostructured system was obtained by the co-encapsulation of $\mathrm{FOH}$ and miconazole nitrate within chitosan nanoparticles and successfully tested against $C$. albicans infection. Chitosan nanoparticles containing the active components were prepared by ionic gelation and showed favourable characteristics for use on mucous membranes. The efficiency of the drug encapsulation was maintained for at least 30 days. The nanoparticles were considered nontoxic in tests with cultured fibroblasts (BALB/c 3T3). Farnesol was capable of inhibiting the yeast to hyphae transition at levels $\geq 300 \mu \mathrm{M}$. The nanoparticles showed antifungal activity against $C$. albicans. The minimal inhibitory concentration of nanoparticles containing miconazole and FOH was slightly lower $(2 \mu \mathrm{g} / \mathrm{mL})$ than the MIC for nanoparticles with only miconazole $(2.5 \mu \mathrm{g} / \mathrm{mL})$. The chitosan nanoparticles containing farnesol not only inhibited fungal proliferation, but also inflammation in the tissue. 


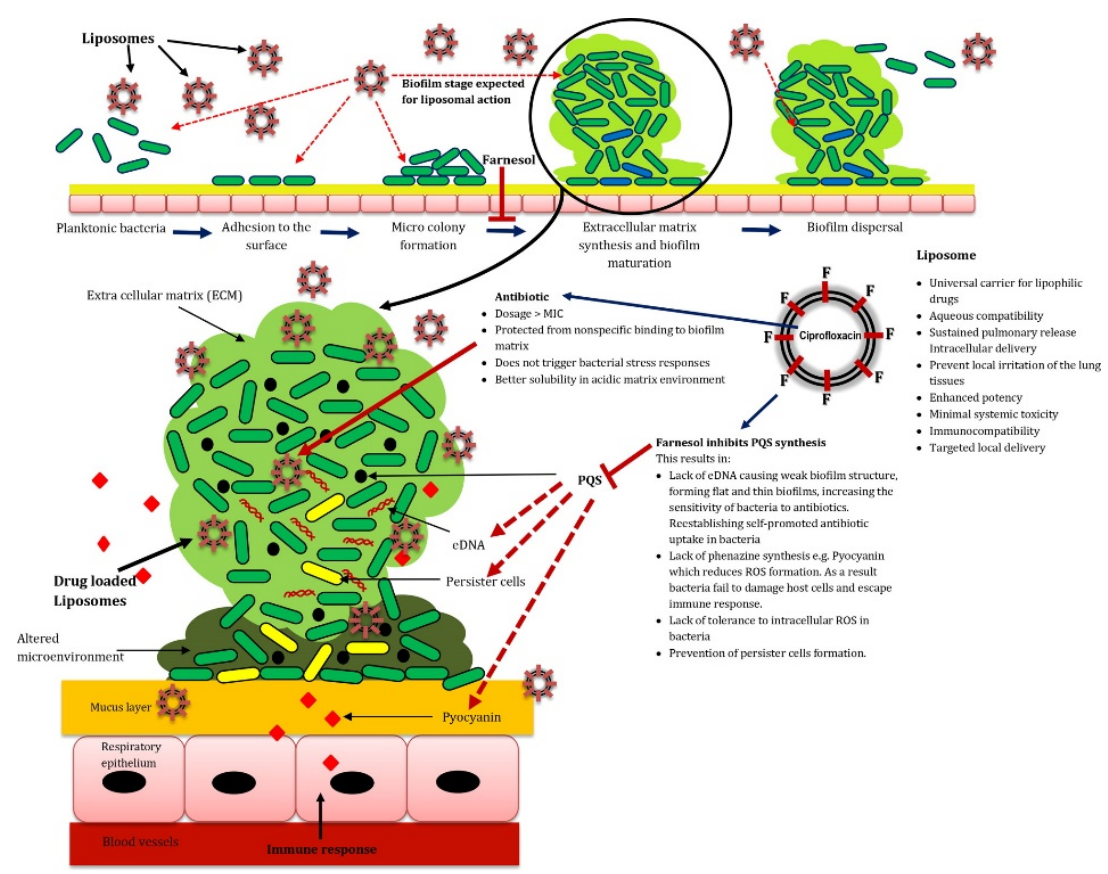

Figure 4. Proposed mechanism explaining the P. aeruginosa biofilm disruption by liposomes loaded with farnesol and ciprofloxacin [80]. Reprinted with permission from (Mol. Pharmaceutics). Copyright (2016) American Chemical Society. https://pubs.acs.org/doi/10.1021/acs.molpharmaceut.6b00360. Further permissions related to the material excerpted should be directed to the ACS.

Nanogels synthesized from natural based polymers can also work as nanocarriers for $\mathrm{FOH}$ and may be designed for pharmaceutical applications. Antifungal properties have been evaluated for nanogels (diameter of 42-70 $\mathrm{nm}$ ) synthetized with alginate (AL) and chitosan (CS) polymers loaded with farnesol [82]. The nanocarriers which contained $300 \mathrm{mM}$ of farnesol influenced the bacterial biofilm growth and HWP1, SAP6 and Rim101 gene expression pattern in C. albicans. The chitosan nanogel released $58 \%$ of farnesol during in vitro studies, and showed larger inhibitory zones than the AL nanogel (37\% FOH release). No significant difference between the control and treatment groups was observed in the cytotoxicity assay $(p>0.05)$. The expression of the HWP1 and SAP6 genes in C. albicans ATCC10231 treated with the CS nanogel (assessed using real-time polymerase chain reaction, PCR) was significantly decreased $(p<0.01)$. The inhibition of key genes contributing to the hyphal formation by the CS/farnesol nanogel suggest that this approach may be a suitable drug delivery strategy.

\subsection{Skin Reparative Therapies and Transdermal Treatment}

Farnesol was found to be a potential skin quality-improving component applicable for reparative and preventive care purposes for UV-damaged skin. FOH containing formulations composed of hydroxypropyl methylcellulose (HPMC) with the addition of hyaluronan (HA) and xanthan gum (XG) were evaluated for the screening of UVB-light and $\mathrm{H}_{2} \mathrm{O}_{2}$ elimination in normal fibroblasts [83]. Gels containing farnesol exhibited anti-photoaging and reparative effects against UVB-induced skin damage (collagen production increase and inflammation alleviation) as well as UVB-screening properties. The best effects were obtained for the gel containing $0.0025 \% \mathrm{FOH}, 0.5 \% \mathrm{HA}, 0.5 \% \mathrm{XG}$ and $2 \%$ HPMC. Further studies have shown that a farnesol-containing $(0.3$ and $0.8 \mathrm{mM})$ gel consisting of $0.1 \%$ HA and $2 \%$ HPMC was used to prepare facial masks with ultraviolet B-screening and skin-repairing capabilities [84]. The collagen production enhancement by skin fibroblasts in vitro was evaluated and an increase in skin smoothness was observed in vivo upon the application of those formulations on UVB-caused sunburnt rat skin. Skin inflammation was alleviated, and interleukin (IL)-6 was decreased in the formulation containing $0.8 \mathrm{mM}$ of $\mathrm{FOH}$. 
Liposomal farnesol can help even more to promote sunburn wound healing. For example, FOH encapsulated in liposomes and admixed into a HPMC gel was studied as a tissue-repairing agent for third-degree burns [85]. In vitro results revealed that liposomal farnesol at intermediate concentrations (from 0.04 to $0.8 \mathrm{mM}$ ) enhanced the collagen production by murine skin fibroblasts. High $(>0.8 \mathrm{mM})$ and low $(<0.04 \mathrm{mM})$ concentrations supressed skin fibroblast proliferation.

A farnesol/alcohol mixture was also used as a permeation enhancer to a propranolol hydrochloride (PPL-HCl)-containing HPMC gel, used in the transdermal treatment for superficial infantile hemangioma (IH) [86]. Farnesol plus isopropanol was the most effective combination that increased the cumulative penetration of PPL- $\mathrm{HCl}\left(\mathrm{Q} 24,6027.4 \pm 563.1 \mu \mathrm{g} / \mathrm{cm}^{2}, \mathrm{ER}, 6.8\right)$, which was significantly higher than that of the control gel $(p<0.05)$.

\section{Conclusions}

The encapsulation of farnesol in polymeric micelles or loading it into macromolecular nanogels increases its stability and antibiofilm activity due to the extended contact time. This approach may also improve interactions of farnesol with planktonic cells, owing to its better dispersion in aqueous systems. The activity of farnesol as an adjuvant enhances the therapy effectiveness for other drugs. Polymeric systems containing the terpenoid alcohol are also a promising solution for skin reparative therapies, not only exhibiting antibiofilm properties and reparative effects, but also UVB screening. Although drug delivery systems based on macromolecular materials may offer a solution to many problems and a significant improvement of the therapeutic effects, one should also be aware of the limitations of these methods. Several synthetic steps may be involved to achieve the designed structure, which is crucial for $\mathrm{pH}$-sensitive materials, and the resulting products need to have appropriate physical properties. It is thus necessary to focus on the balance between the pros and the cons as well as to find a solution to those problems in future research.

Author Contributions: All authors contributed equally to this manuscript. All authors have read and agreed to the published version of the manuscript.

Funding: Maria Nowacka thanks the Centre of Molecular and Macromolecular Studies, Polish Academy of Sciences for support under the statutory funds and Young Researchers Development program.

Conflicts of Interest: The authors declare no conflict of interest.

\section{References}

1. Polke, M.; Jacobsen, I.D. Quorum sensing by farnesol revisited. Curr. Genet. 2017, 63, 791-797. [CrossRef] [PubMed]

2. Zawrotniak, M.; Wojtalik, K.; Rapala-Kozik, M. Farnesol, a Quorum-sensing molecule of Candida albicans triggers the release of neutrophil extracellular traps. Cells 2019, 8, 1611. [CrossRef] [PubMed]

3. Ahmed, S.A.K.S.; Rudden, M.; Smyth, T.J.; Dooley, J.S.G.; Marchant, R.; Banat, I.M. Natural quorum sensing inhibitors effectively downregulate gene expression of Pseudomonas aeruginosa virulence factors. Appl. Microbiol. Biotechnol. 2019, 103, 3521-3535. [CrossRef] [PubMed]

4. Dancewicz, K.; Gliszczynska, A.; Halarewicz, A.; Wawrzenczyk, C.; Gabrys, B. Effect of farnesol and its synthetic derivatives on the settling behaviour of the peach potato aphid Myzus persicae (Sulz.). Pestycydy 2010, 1-4, 51-57.

5. Jamalian, A.; Shams-Ghahfarokhi, M.; Jaimand, K.; Pashootan, N.; Amani, A.; Razzaghi-Abyaneh, M.J. Chemical composition and antifungal activity of Matricaria recutita flower essential oil against medically important dermatophytes and soil-borne pathogens. Mycol. Med. 2012, 22, 308-315. [CrossRef]

6. Wroblewska-Kurdyk, A.; Dancewicz, K.; Gliszczynska, A.; Gabrys, B. New insight into the behaviour modifying activity of two natural sesquiterpenoids farnesol and nerolidol towards Myzus persicae (Sulzer) (Homoptera: Aphididae). Bull. Entomol. Res. 2020, 110, 249-258. [CrossRef]

7. Gupta, P.; Sharma, M.; Arora, N.; Pruthi, V.; Poluri, K.M. Chemistry and Biology of Farnesol and its derivatives: Quorum sensing molecules with immense therapeutic potential. Curr. Top. Med. Chem. 2018, 18, 1937-1954. [CrossRef] 
8. De Matos, S.P.; Teixeira, H.F.; de Lima, Á.A.N.; Veiga-Junior, V.F.; Koester, L.S. Essential oils and isolated terpenes in nanosystems designed for topical administration: A Review. Biomolecules 2019, 9, 138. [CrossRef]

9. Vitiello, G.; Silvestri, B.; Luciani, G. Learning from nature: Bioinspired strategies towards antimicrobial nanostructured systems. Curr. Top. Med. Chem. 2018, 18, 22-41. [CrossRef]

10. Crick, D.C.; Andres, D.A.; Waechter, C.J. Farnesol is utilized for protein isoprenylation and the biosynthesis of cholesterol in mammalian cells. Biochem. Biophys. Res. Commun. 1995, 211, 590-599. [CrossRef]

11. Hornby, J.M.; Jensen, E.C.; Lisec, A.D.; Tasto, J.J.; Jahnke, B.; Shoemaker, R.; Dussault, P.; Nickerson, K.W. Quorum sensing in the dimorphic fungus Candida albicans is mediated by farnesol. Appl. Environ. Microbiol. 2001, 67, 2982-2992. [CrossRef] [PubMed]

12. Kong, E.F.; Tsui, C.; Kucharíková, S.; Van Dijck, P.; Jabra-Rizk, M.A. Modulation of Staphylococcus aureus response to antimicrobials by the Candida albicans quorum sensing molecule farnesol. Antimicrob. Agents Chemother. 2017, 61, e01573-17. [CrossRef] [PubMed]

13. Shchepin, R.; Hornby, J.M.; Burger, E.; Niessen, T.; Dussault, P.; Nickerson, K.W. Quorum sensing in Candida albicans: Probing farnesol's mode of action with 40 natural and synthetic farnesol analogs. Chem. Biol. 2003, 10, 743-750. [CrossRef]

14. Rodriques, C.F.; Černáková, L. Farnesol and Tyrosol: Secondary metabolites with a crucial quorum-sensing role in Candida biofilm development. Genes 2020, 11, 444. [CrossRef] [PubMed]

15. Sato, T.; Watanabe, T.; Mikami, T.; Matsumoto, T. Farnesol, a morphogenetic autoregulatory substance in the dimorphic fungus Candida albicans, inhibits hyphae growth through suppression of a mitogen-activated protein kinase cascade. Biol. Pharm. Bull. 2004, 27, 751-752. [CrossRef]

16. Davis-Hanna, A.; Piispanen, A.E.; Stateva, L.I.; Hogan, D.A. Farnesol and dodecanol effects on the Candida albicans Ras1-cAMP signalling pathway and the regulation of morphogenesis. Mol. Microbiol. 2008, 67, 47-62. [CrossRef]

17. Zakikhany, K.; Naglik, J.R.; Schmidt-Westhausen, A.; Holland, G.; Schaller, M.; Hube, B. In vivo transcript profiling of Candida albicans identifies a gene essential for interepithelial dissemination. Cell Microbiol. 2007, 9, 2938-2954. [CrossRef]

18. Martin, R.; Moran, G.P.; Jacobsen, I.D.; Heyken, A.; Domey, J.; Sullivan, D.J.; Kurzai, O.; Hube, B. The Candida albicans-specific gene EED1encodes a key regulator of hyphal extension. PLoS ONE 2011, 6, e18394. [CrossRef]

19. Nickerson, K.W.; Atkin, A.L. Deciphering fungal dimorphism: Farnesol's unanswered questions. Mol. Microbiol. 2017, 103, 567-575. [CrossRef]

20. Nickerson, K.W.; Atkin, A.L.; Hornby, J.M. Quorum sensing in dimorphic fungi: Farnesol and beyond. Appl. Environ. Microbiol. 2006, 72, 3805-3813. [CrossRef]

21. Sebaa, S.; Boucherit-Otmani, Z.; Courtois, P. Effects of tyrosol and farnesol on Candida albicans biofilm. Mol. Med. Rep. 2019, 19, 3201-3209. [CrossRef] [PubMed]

22. Cao, Y.Y.; Cao, Y.B.; Xu, Z.; Ying, K.; Li, Y.; Xie, Y.; Zhu, Z.Y.; Chen, W.S.; Jiang, Y.Y. cDNA microarray analysis of differential gene expression in Candida albicans biofilm exposed to farnesol. Antimicrob. Agents Chemother. 2005, 49, 584-589. [CrossRef] [PubMed]

23. Chen, S.; Xia, J.; Li, C.; Zuo, L.; Wei, X. The possible molecular mechanisms of farnesol on the antifungal resistance of C. albicans biofilms: The regulation of CYR1 and PDE2. BMC Microbiol. 2018, 18, 203. [CrossRef] [PubMed]

24. Padder, S.A.; Prasad, R.; Shah, A.H. Quorum sensing: A less known mode of communication among fungi. Microbiol. Res. 2018, 210, 51-58. [CrossRef] [PubMed]

25. Jabra-Rizk, M.A.; Meiller, T.F.; James, C.E.; Shirtliff, M.E. Effect of farnesol on Staphylococcus aureus biofilm formation and antimicrobial susceptibility. Agents Chemother. 2006, 50, 1463-1469. [CrossRef] [PubMed]

26. Vila, T.; Kong, E.F.; Ibrahim, A.; Piepenbrink, K.; Shetty, A.C.; McCracken, C.; Bruno, V.; Jabra-Rizk, M.A. Candida albicans quorum-sensing molecule farnesol modulates staphyloxanthin production and activates the thiol-based oxidative-stress response in Staphylococcus aureus. Virulence 2019, 10, 625-642. [CrossRef]

27. Jeon, J.G.; Pandit, S.; Xiao, J.; Greqoire, S.; Falsetta, M.L.; Klein, M.I. Influences of trans-trans farnesol, a membrane-targeting sesquiterpenoid, on Streptococcus mutans physiology and survival within mixed-species oral biofilms. J. Oral Sci. 2011, 3, 98-106. [CrossRef]

28. Pammi, M.; Liang, R.; Hicks, J.M.; Barrish, J.; Versalovic, J. Farnesol decreases biofilms of Staphylococcus epidermidis and exhibits synergy with nafcillin and vancomycin. Pediatr. Res. 2011, 70, 578-583. [CrossRef] 
29. Dixon, E.F.; Hall, R.A. Noisy neighbourhoods: Quorum sensing infungal-polymicrobial infections. Cell. Microbiol. 2015, 17, 1431-1441. [CrossRef]

30. Cugini, C.; Calfee, M.W.; Farrow, J.M., III; Morales, D.K.; Pesci, E.C.; Hogan, D.A. Farnesol, a common sesquiterpene, inhibits PQS production in Pseudomonas aeruginosa. Mol. Microbiol. 2007, 65, 896-906. [CrossRef]

31. Brehm-Stecher, B.F.; Johnson, E.A. Sensitization of Staphylococcus aureus and Escherichia coli to antibiotics by the sesquiterpenoids nerolidol, farnesol, bisabolol, and apritone. Antimicrob. Agents Chemother. 2003, 47, 3357-3360. [CrossRef]

32. Semighini, C.P.; Hornby, J.M.; Dumitru, R.; Nickerson, K.W.; Harris, S.D. Farnesol-induced apoptosis in Aspergillus nidulans reveals a possible mechanism for antagonistic interactions between fungi. Mol. Microbiol. 2006, 59, 753-764. [CrossRef] [PubMed]

33. Wang, X.; Wang, Y.; Zhou, Y.; Wei, X. Farnesol induces apoptosis-like cell death in the pathogenic fungus Aspergillus flavus. Mycologia 2014, 106, 881-888. [CrossRef] [PubMed]

34. Leonhardt, I.; Spielberg, S.; Michael Weber, M.; Albrecht-Eckardt, D.; Bläss, M.; Claus, R.; Barz, C.; Scherlach, K.; Hertweck, C.; Löffler, J.; et al. The fungal quorum-sensing molecule farnesol activates innate immune cells but suppresses cellular adaptive immunity. mBio 2015, 6, e00143-15. [CrossRef] [PubMed]

35. Mosel, D.D.; Dumitru, R.; Hornby, J.M.; Atkin, A.L.; Nickerson, K.W. Farnesol concentrations required to block germ tube formation in Candida albicans in the presence and absence of serum. Appl. Environ. Microbiol. 2005, 71, 4938-4940. [CrossRef] [PubMed]

36. Rodríguez-Hernández, J. Polymers against Microorganisms. In On the Race to Efficient Antimicrobial Materials, 1st ed.; Springer International Publishing AG: Cham, Switzerland, 2017.

37. Nowacka, M.; Rygała, A.; Kregiel, D.; Kowalewska, A. Poly(silsesquioxanes) and poly(siloxanes) grafted with N-acetylcysteine for eradicating mature bacterial biofilms in water environment. Coll. Surf. B Biointerf. 2018, 172, 627-634. [CrossRef]

38. Kręgiel, D.; Rygała, A.; Kolesińska, B.; Nowacka, M.; Herc, A.S.; Kowalewska, A. Antimicrobial and antibiofilm $\mathrm{N}$-acetyl-L-cysteine grafted siloxane polymers with potential for use in water systems. Int. J. Mol. Sci. 2019, 20, 2011. [CrossRef]

39. Santos, M.R.E.; Fonseca, A.C.; Mendonça, P.V.; Branco, R.; Serra, A.C.; Morais, P.V.; Coelho, J.F.J. Recent developments in antimicrobial polymers: A review. Materials 2016, 9, 599. [CrossRef]

40. Huang, K.-S.; Yang, C.-H.; Huang, S.-L.; Chen, C.-Y.; Lu, Y.-Y.; Lin, Y.-S. Recent advances in antimicrobial polymers: A mini-review. Int. J. Mol. Sci. 2016, 17, 1578. [CrossRef]

41. Liu, N.-Y.; Zhu, J.-Y.; Zhang, T.; Dong, S.-L. Characterization of two odorant binding proteins in Spodoptera exigua reveals functional conservation and difference. Comp. Biochem. Phys. A 2017, 213, 20-27. [CrossRef]

42. Kim, C.; Kim, H. Synthesis of Farnesyl-Terminated Carbosilane Dendrimer. Synthesis 2005, 3, 381-386. [CrossRef]

43. Parker, D.J.; Jones, H.A.; Petcher, S.; Cervini, L.; Griffin, J.M.; Akhtarb, R.; Hasell, T. Low cost and renewable sulfur-polymers by inverse vulcanisation, and their potential for mercury capture. J. Mater. Chem. A 2017, 5, 11682-11692. [CrossRef]

44. Manteghi, A.; Ahmadi, S.; Arabi, H. Polyolefin elastomer grafted unsaturated hindered phenol esters: Synthesis and antioxidant behavior. Des. Monomers Polym. 2016, 19, 569-576. [CrossRef]

45. Gómez-Estaca, J.; López-de-Dicastillo, C.; Hernández-Muñoz, P.; Catalá, R.; Gavara, R. Advances in antioxidant active food packaging. Trends Food Sci. Technol. 2014, 35, 42-51. [CrossRef]

46. Zhang, Y.; Li, H.; Zhang, Y.; Li, Q.; Ma, Z.; Dong, J.Y.; Hu, Y. Synthesis and properties of polyethylene-bound antioxidants. Macromol. Chem. Phys. 2014, 215, 763-775. [CrossRef]

47. Laschke, M.W.; Strohe, A.; Scheuer, C.; Eglin, D.; Verrier, S.; Alini, M.; Pohlemann, T.; Menger, M.D. In vivo biocompatibility and vascularization of biodegradable porous polyurethane scaffolds for tissue engineering. Acta Biomaterialia 2009, 5, 1991-2001. [CrossRef]

48. O’Brien, F.J. Biomaterials \& scaffolds for tissue engineering. Mater. Today 2011, 14, 88-95.

49. Eglin, D.; Grad, S.; Gogolewski, S.; Alini, M. Farsenol-modified biodegradable polyurethanes for cartilage tissue engineering. J. Biomed. Mater. Res. A. 2010, 92, 393-408. [CrossRef]

50. Hawser, S.P.; Douglas, L.J. Biofilm formation by Candida species on the surface of catheter materials in vitro. Infect. Immun. 1994, 62, 915-921. [CrossRef]

51. Deveau, A.; Hogan, D.A. Linking quorum sensing regulation and biofilm formation by Candida albicans. Methods Mol. Biol. 2011, 692, 219-233. 
52. Bhattacharyya, S.; Agrawal, A.; Knabe, C.; Ducheyne, P. Sol gel silica controlled release thin films for the inhibition of methicillin-resistant Staphylococcus aureus. Biomaterials 2014, 35, 509-517. [CrossRef] [PubMed]

53. Reynolds, P.E. Structure, biochemistry and mechanism of action of glycopeptide antibiotics. Eur. J. Clin. Microbiol. Infect. Dis. 1989, 8, 943-950. [CrossRef] [PubMed]

54. Schilling, A.; Neuner, E.; Rehm, S.J. Vancomycin: A 50-something-year-old antibiotic we still don't understand. Cleve. Clin. J. Med. 2011, 78, 465-471. [CrossRef] [PubMed]

55. Gomes, F.; Leite, B.; Teixeira, P.; Cerca, N.; Azeredo, J.; Oliveira, S. Farnesol as antibiotics adjuvant in Staphylococcus epidermidis control in vitro. Am. J. Med. Sci. 2011, 341, 191-195. [CrossRef] [PubMed]

56. Bhadani, A.; Rane, J.; Veresmortean, C.; Banerjee, S.; John, G. Bio-inspired surfactants capable of generating plant volatiles. Soft Matter. 2015, 11, 3076-3082. [CrossRef] [PubMed]

57. Tse, G.; Blankschtein, D.; Shefer, A.; Shefer, S. Thermodynamic prediction of active ingredient loading in polymeric microparticles. J. Controll. Release 1999, 60, 77-100. [CrossRef]

58. Sousa, F.L.; Horta, S.; Santos, M.; Rocha, S.M.; Trindade, T. Release behavior of trans,trans-farnesol entrapped in amorphous silica capsules. Results Pharma Sci. 2012, 2, 52-56. [CrossRef]

59. Sousa, F.L.; Santos, M.; Rocha, S.M.; Trindade, T. Encapsulation of essential oils in $\mathrm{SiO}_{2}$ microcapsules and release behaviour of volatile compounds. J. Microencapsul. 2014, 7, 627-635. [CrossRef]

60. Singh, R.; Smitha, M.S.; Singh, S.P. The role of nanotechnology in combating multi-drug resistant bacteria. J. Nanosci. Nanotechnol. 2014, 14,1-12. [CrossRef]

61. Qayyum, S.; Khan, A.U. Nanoparticles vs. biofilms: A battle against another paradigm of antibiotic resistance. Med. Chem. Commun. 2016, 7, 1479-1498. [CrossRef]

62. Liu, Y.; Shi, L.; Su, L.; van der Mei, H.C.; Jutte, P.C.; Ren, Y.; Busscher, H.J. Nanotechnology-based antimicrobials and delivery systems for biofilm-infection control. Chem. Soc. Rev. 2019, 48, 428-446. [CrossRef]

63. Chen, F.; Liu, X.-M.; Rice, K.C.; Li, X.; Yu, F.; Reinhardt, R.A.; Bayles, K.W.; Wang, D. Tooth-binding micelles for dental caries prevention. Antimicrob. Agents Chemother. 2009, 53, 4898-4902. [CrossRef]

64. Mogen, A.B.; Chen, F.; Ahn, S.-J.; Burne, R.A.; Wang, D.; Rice, K.C. Pluronics-formulated farnesol promotes efficient killing and demonstrates novel interactions with Streptococcus mutans Biofilms. PLoS ONE 2015, 10, e0133886. [CrossRef]

65. Canaparo, R.; Foglietta, F.; Giuntini, F.; Della Pepa, C.; Dosio, F.; Serpe, L. Recent developments in antibacterial therapy: Focus on stimuli-responsive drug-delivery systems and therapeutic nanoparticles. Molecules 2019, 24, 1991. [CrossRef]

66. Abraham, T.; Mao, M.; Tan, C. Engineering approaches of smart, bio-inspired vesicles for biomedical applications. Phys. Biol. 2018, 15, 061001. [CrossRef]

67. Alvarez-Lorenzo, C.; Garcia-Gonzalez, C.A.; Bucio, E.; Concheiro, A. Stimuli-responsive polymers for antimicrobial therapy: Drug targeting, contact killing surfaces and competitive release. Expert Opin. Drug Deliv. 2016, 13, 1109-1119. [CrossRef]

68. Benoit, D.S.W.; Sims, K.R., Jr.; Fraser, D. Nanoparticles for oral biofilm treatments. ACS Nano 2019, 13, 4869-4875. [CrossRef]

69. Hu, C.; Wang, L.-L.; Lin, Y.-Q.; Liang, H.-M.; Zhou, S.-Y.; Zheng, F.; Feng, X.-L.; Rui, Y.-Y.; Shao, L.-Q. Nanoparticles for the treatment of oral biofilms: Current state, mechanisms, influencing factors, and prospects. Adv. Healthcare Mater. 2019, 8, 1901301. [CrossRef]

70. Horev, B.; Klein, M.I.; Hwang, G.; Li, Y.; Kim, D.; Koo, H.; Benoit, D.S.W. pH-activated nanoparticles for controlled topical delivery of farnesol to disrupt oral biofilm virulence. ACS Nano 2015, 9, 2390-2404. [CrossRef]

71. Zhou, J.; Horev, B.; Hwang, G.; Klein, M.I.; Koo, H.; Benoit, D.S.W. Characterization and optimization of pH responsive polymer nanoparticles for drug delivery to oral biofilms. J. Mater. Chem. B 2016, 4, 3075-3085. [CrossRef]

72. Yi, Y.; Wang, L.; Chen, L.; Lin, Y.; Luo, Z.; Chen, Z.; Li, T.; Wu, J.; Zhong, Z. Farnesal-loaded pH-sensitive polymeric micelles provided effective prevention and treatment on dental caries. J. Nanobiotechnol. 2020. [CrossRef]

73. Dora, C.P.; Singh, S.K.; Kumar, S.; Datusalia, A.K.; Deep, A. Development and characterization of nanoparticles of glibenclamide by solvent displacement method. Acta Pol. Pharm. 2010, 67, 283-290. [PubMed] 
74. Sims, K.R., Jr.; Liu, Y.; Hwang, G.; Jung, H.I.; Koo, H.; Benoit, D.S.W. Enhanced design and formulation of nanoparticles for anti-biofilm drug delivery. Nanoscale 2019, 11, 219-236. [CrossRef]

75. Barot, T.; Rawtani, D.; Kulkarni, P.; Hussain, C.M.; Akkireddy, S. Physicochemical and biological assessment of flowable resin composites incorporated with farnesol loaded halloysite nanotubes for dental applications. J. Mech. Behav. Biomed. Mater. 2020, 104, 103675. [CrossRef]

76. Cavallaro, G.; Chiappisi, L.; Pasbakhsh, P.; Gradzielski, M.; Lazzara, G. A structural comparison of halloysite nanotubes of different origin by Small-Angle Neutron Scattering (SANS) and Electric Birefringence. Appl. Clay Sci. 2018, 160, 71-80. [CrossRef]

77. Pandey, G.; Munguambe, D.M.; Tharmavaram, M.; Rawtani, D.; Agrawal, Y.K. Halloysite nanotubes-An efficient 'nano-support' for the immobilization of $\alpha$-amylase. Appl. Clay Sci. 2017, 136, 184-191. [CrossRef]

78. De Castilho, A.R.F.; Rosalen, P.L.; de Souza Araújo, I.J.; Kitagawa, I.L.; de Araujo Costa, C.A.; Janal, M.N.; Alves, M.C.; Duarte, S.; Filho, P.N.L.; Stipp, R.N.; et al. Trans,trans-farnesol, an antimicrobial natural compound, improves glass ionomer cement properties. PLoS ONE 2019, 14, e0220718. [CrossRef]

79. Chávez-Andrade, G.-M.; Tanomaru-Filho, M.; Basso Bernardi, M.I.; de Toledo Leonardo, R.; Faria, G.; Guerreiro-Tanomaru, J.M. Antimicrobial and biofilm anti-adhesion activities of silver nanoparticles and farnesol against endodontic microorganisms for possible application in root canal treatment. Arch. Oral Biol. 2019, 107, 104481. [CrossRef]

80. Bandara, H.M.H.N.; Herpin, M.J.; Kolacny, D., Jr.; Harb, A.; Romanovicz, D.; Smyth, H.D.C. Incorporation of farnesol significantly increases the efficacy of liposomal Ciprofloxacin against Pseudomonas aeruginosa biofilms in vitro. Mol. Pharm. 2016, 13, 2760-2770. [CrossRef]

81. Costa, A.F.; Araujo, D.E.; Cabral, M.S.; Brito, I.T.; De Menezes Leite, L.B.; Pereira, M.; Amaral, A.C. Development, characterization, and in vitro-in vivo evaluation of polymeric nanoparticles containing miconazole and farnesol for treatment of vulvovaginal candidiasis. Med. Mycol. 2019, 57, 52-62. [CrossRef]

82. Nikoomanesh, F.; Roudbarmohammadi, S.; Khoobi, M.; Haghighi, F.; Roudbary, M. Design and synthesis of mucoadhesive nanogel containing farnesol: Investigation of the effect on HWP1, SAP6 and Rim101 genes expression of Candida albicans in vitro. Artif. Cell. Nanomed. Biotechnol. 2019, 47, 64-72. [CrossRef]

83. Wu, G.X.; Huang, H.H.; Chang, H.R.; Kuo, S.M. Evaluation of the UVB-screening capacity and restorative effects exerted by farnesol gel on UVB-caused sunburn. Environ. Toxicol. 2018, 33, 488-507. [CrossRef] [PubMed]

84. Huang, Y.W.; Huang, H.H.; Wu, G.X.; Chang, H.R.; Wu, K.L.; Kuo, S.M. Antiaging and smoothness-improving properties of farnesol-based facial masks on rat skin exposed to ultraviolet B. J. Cosmet. Dermatol. 2020, 19, 540-552. [CrossRef]

85. Wu, Y.C.; Wu, G.X.; Huang, H.H.; Shyh Ming Kuo, S.M. Liposome-encapsulated farnesol accelerated tissue repair in third-degree burns on a rat model. Burns 2019, 45, 1139-1151. [CrossRef] [PubMed]

86. Zhou, W.; He, S.; Yang, Y.; Jian, D.; Chen, X.; Ding, J. Formulation, characterization and clinical evaluation of propranolol hydrochloride gel for transdermal treatment of superficial infantile hemangioma. Drug Dev. Ind. Pharm. 2015, 41, 1109-1119. [CrossRef]

(C) 2020 by the authors. Licensee MDPI, Basel, Switzerland. This article is an open access article distributed under the terms and conditions of the Creative Commons Attribution (CC BY) license (http://creativecommons.org/licenses/by/4.0/). 